\title{
Physical activity and health in adolescence
}

\author{
Authors: Bhavesh Kumar, ${ }^{A}$ Rebecca Robinson ${ }^{B}$ and Simon Till ${ }^{C}$
}

Adolescence represents a critical period of development during which personal lifestyle choices and behaviour patterns establish, including the choice to be physically active. Physical inactivity, sedentary behaviour and low cardiorespiratory fitness are strong risk factors for the development of chronic diseases with resulting morbidity and mortality, as well as economic burden to wider society from health and social care provision, and reduced occupational productivity. Worrying trends in adverse physical activity behaviours necessitate urgent and concerted action. Healthcare professionals caring for adolescents and young adults are ideally placed and suited to deliver powerful messages promoting physical activity and behaviour change. Every encounter represents an opportunity to ask about physical activity, provide advice, or signpost to appropriate pathways or opportunities. Key initial targets include getting everyone to reduce their sedentary behaviour and be more active, with even a little being more beneficial than none at all.

KEYWORDS: Physical activity, physical inactivity, sedentary behaviour, cardiorespiratory fitness, adolescence, young adults, health promotion, chronic disease

There is overwhelming evidence for the lifelong health benefits of a physically active lifestyle and the health risks of inactivity and sedentary behaviour. ${ }^{1}$ England's Chief Medical Officer has acknowledged that the benefits of regular physical activity on health, longevity, and wellbeing 'easily surpass the effectiveness of any drugs or other medical treatment'.

The process of cardiovascular disease (CVD) begins in childhood, and associated risk factors, including inactivity and obesity, track through adolescence (ages 11-25 years ${ }^{3}$ ) into adulthood, imparting heightened risk of premature mortality. Since physical activity (PA) patterns are established during

Authors: ${ }^{\text {A }}$ consultant physician in sport and exercise medicine, Institute of Sport Exercise and Health, University College London,

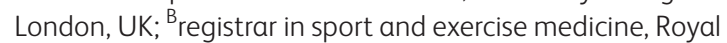

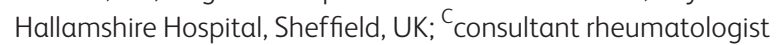
and reader in physical activity medicine, Sheffield Teaching Hospitals and Sheffield Hallam University, Sheffield, UK childhood and adolescence, every consultation represents an opportunity to promote PA.

\section{The burden of physical inactivity and obesity}

In England, only $21 \%$ of boys and $16 \%$ of girls meet minimum PA recommendations for their health ${ }^{4}$ (Table 1), a trend that remains in decline. ${ }^{5}$ Half of the UK's population of 11-25 year olds ( 4.5 million) are inactive. ${ }^{6}$ Those from lower socioeconomic groups and ethnic minorities have less access to environmentally safe PA and sports. ${ }^{7}$

The proportion of active individuals declines significantly in adolescence (Table 2), with girls less active than boys (56 vs $39 \%$ ). This tallies with the continued rise in obesity rates among $11-15$ year olds, with $38 \%$ being overweight. ${ }^{8}$

Increased self-consciousness and peer pressure through adolescence and puberty may be factors in declining PA involvement and therefore timely, sensitive engagement with this group is essential.

$50-75 \%$ of obese children and adolescents will become obese adults with associated higher rates of metabolic, vascular and musculoskeletal disease. ${ }^{9-11}$ Obesity increases the risk of type-2 diabetes five-fold in men and over ten-fold in women, triples the risk of cardiovascular disease and colon cancer, and increases risk of liver disease and osteoarthritis. ${ }^{4}$

The economic burden of physical inactivity (PIA) in England is immense. The lifetime cost to the UK economy is an

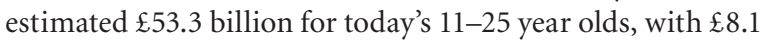
billion directed towards care for inactivity-related disease. Impact from reduced quality of life and life expectancy costs around $\mathfrak{£} 10,000$ per child. ${ }^{6}$

Table 1. The recommended 'dose' of PA in adolescents. $^{2}$

\begin{tabular}{ll}
$\begin{array}{l}\text { Exercise prescription } \\
\text { using FITT }\end{array}$ & $\begin{array}{l}\text { PA guidelines for young people } \\
\text { aged 5-18 years }\end{array}$ \\
Frequency & Daily \\
Intensity & $\begin{array}{l}\text { Moderate to vigorous } \\
\text { All PA, exercise or sport. Muscle } \\
\text { Type }\end{array}$ \\
& $\begin{array}{l}\text { activity three times weekly } \\
\text { At least } 60 \text { minutes daily. Minimise } \\
\text { prolonged sedentary time }\end{array}$ \\
Time & \\
\hline PA= physical activity. &
\end{tabular}


Table 2. Proportion of children and adolescents meeting current UK PA guidelines. ${ }^{4}$

\begin{tabular}{lll} 
Age group, years & \multicolumn{2}{l}{ Percentage meeting guidelines, \% } \\
\cline { 2 - 3 } & Males & Females \\
$5-7$ & 24 & 23 \\
$13-15$ & 14 & 8 \\
\hline
\end{tabular}

$\mathrm{PA}=$ physical activity

\section{Physical activity behaviours and health risk}

Physiologically, there exists a complex dynamic between the 'dose' of regular PA undertaken, level of sedentariness, body mass index (BMI), fitness and the link with non-communicable disease. Box 1 explains some of the terminology relating to these key concepts.

PIA is the fourth leading cause of global mortality. ${ }^{1}$ PA plays a key role in developing healthy cardiorespiratory fitness (CRF), which in turn correlates inversely with morbidity and mortality. However, within groups with similar fitness, the risk is higher for inactive individuals compared with those meeting PA guidelines. Furthermore, active overweight and obese people have around $50 \%$ lower non-communicable disease risk compared with unfit normal weight counterparts.

If everyone had a 'moderate' level of CRF, overall mortality would be reduced by about $17 \%$, whereas if no one were obese, the risk reduction would be only $2-3 \% .{ }^{10}$ It is therefore imperative to increase both PA and CRF levels to offset the adverse effects of inactivity, sedentary time and associated obesity.

The challenging reality is the common co-presentation of PIA and obesity. Current guideline PA levels for adolescents ${ }^{4}$ remain insufficient to offset weight gain associated with today's sedentary lifestyles, highlighting the need for promotion of an active lifestyle and healthy diet. ${ }^{12}$ The inactive adolescent with normal BMI is at heightened risk of chronic disease relative to an overweight, active peer.

\section{Sedentary lifestyles and adolescent health}

Public Health England (PHE) advise that all children and young people should minimise sedentary time. ${ }^{13}$

Sedentary behaviour is an independent cause of chronic disease and one in six deaths in the UK. This cluster of behaviours, characterised by very low energy expenditure, is highly prevalent in modern society. The adolescent playing sport for an hour three times per week is still likely to lead a 'sedentary' lifestyle relative to their peers 50 years ago. ${ }^{14}$ Young adolescents spend an average of 6.1 hours each day watching television, using a computer or other smart technology, referred to as screen time. A child born in 2012 will accrue three years of screen time by age 18 . Screen time is positively associated with obesity, CVD and mortality. ${ }^{15}$

Studies indicate continuous periods of sedentariness pose more potent health risks than obesity. Independent of BMI, the most physically active people reduce their risk of developing CVD by $40 \%$, compared with the most sedentary. ${ }^{16}$ Engaging in moderate and vigorous PA at current guideline levels without further interrupting sedentary behaviour is likely to be insufficient to offset health risks. ${ }^{17}$

Advancing technology which is associated with sedentary behaviour compounds the challenges of PIA. While current recommendations to limit screen time may be unrealistic, paradoxically there may be opportunity to engage young people in PA through technology, for example with apps and virtual training partners. Wearable technology within smartphones, watches and tablets can monitor PA levels and track biometric data.

\section{Box 1. Glossary of PA terms.}

> PA: Any body movement that increases energy expenditure. Includes activities of daily living, occupation, active transport, play, exercise and sport.

> Exercise: Planned, structured, repetitive body movement undertaken to improve physical fitness.

Physical fitness: Ability to perform physical activity. Requires physiological health and neuromotor skills.

CRF: Ability of the body's circulatory and respiratory systems to supply fuel and oxygen during sustained PA.

$>$ PIA: Insufficient amount of PA according to Department of Health guidelines. ${ }^{10}$

> METs: A measure of energy expenditure. The amount of oxygen required for a given activity in relation to sitting (1 MET). $1 \mathrm{MET}$ varies between individuals, though a mean approximation is $3.5 \mathrm{ml} \mathrm{O} / \mathrm{kg} / \mathrm{min}=1 \mathrm{kcal} / \mathrm{kg} / \mathrm{h}$

> Sedentary behaviour: Very low energy expenditure (<1 MET) eg TV viewing, computer use, motorised transport.

> Light-intensity PA: 1-3 METs; sustained PA where heart rate, respiratory rate and body temperature rise above resting levels (eg standing, strolling).

> Moderate-intensity PA: 3-6 METs. Able to talk but not sing (eg brisk walking, steady pace cycling).

> Vigorous-intensity PA: 6-9 METs (eg running, shovelling).

> Very vigorous PA: >9 METs (eg squash, cross-country skiing, stair running).

> Moderate and vigorous PA: most beneficial for health at population level.

CRF = cardiorespiratory fitness; METs = metabolic equivalent; PA = physical activity; PIA = physical inactivity. 


\section{Box 2. The health benefits of PA in adolescence. ${ }^{18,19}$}

> Optimising muscular strength and flexibility

$>$ Maintaining healthy weight

> Attaining peak bone mass

Developing cardiovascular health

Developing neuromuscular awareness

Benefiting cognition and mental health, mood, sleep and academic attainment

> Improving wellbeing and social behaviour

$\mathrm{PA}=$ physical activity.

\section{Health benefits of physical activity}

PA is potent medicine that promotes healthy development in adolescence. Box 2 summarises some of the key benefits.

There is a clear inverse correlation between PA and all-cause mortality. Regular PA in youth improves cardiovascular physiology, including blood pressure, lipid profile, insulin sensitivity and endothelial function. Furthermore, the resulting higher calorie expenditure helps weight control. ${ }^{12}$

Weight-bearing and resistance exercise play vital roles in developing muscle mass and attaining peak bone density in early adulthood, important for musculoskeletal health in later life.

PA increases cerebral blood flow and circulating levels of norepinephrine and endorphins, positively affecting mood, self-confidence and concentration, while moderating anxiety, stress and anger. Cognitive development, skill acquisition and behaviour are also improved. ${ }^{18}$

Sport participation can help develop social skills, teamwork and leadership. PA is associated with reduced levels of risk-taking behaviours, smoking and excessive alcohol consumption. $^{20}$

\section{Every healthcare professional's role}

The healthcare professional is ideally placed to engage adolescents in healthy lifestyle choices involving optimal PA to improve health and wellbeing. Healthcare professionals can also actively engage families, schools and local authorities to support young people in an active environment.

Every patient encounter is an opportunity to elicit PA and sedentary behaviour levels, and offer advice. This should be recorded within the social history, documenting daily minutes engaged in moderate and vigorous PA and time spent sedentary. Information and advice offered to the adolescent must be relatable in context of their lifestyle and any coexistent medical conditions.

We advocate a PA 'prescription' for all young people within their healthcare plan. A useful tool for providing specific advice is the FITT principle. Table 1 demonstrates how current guideline levels of PA can be prescribed according to FITT. Fig 1 illustrates a model for integrating PA within the consultation and Table 3 provides key strategies for successful PA promotion.

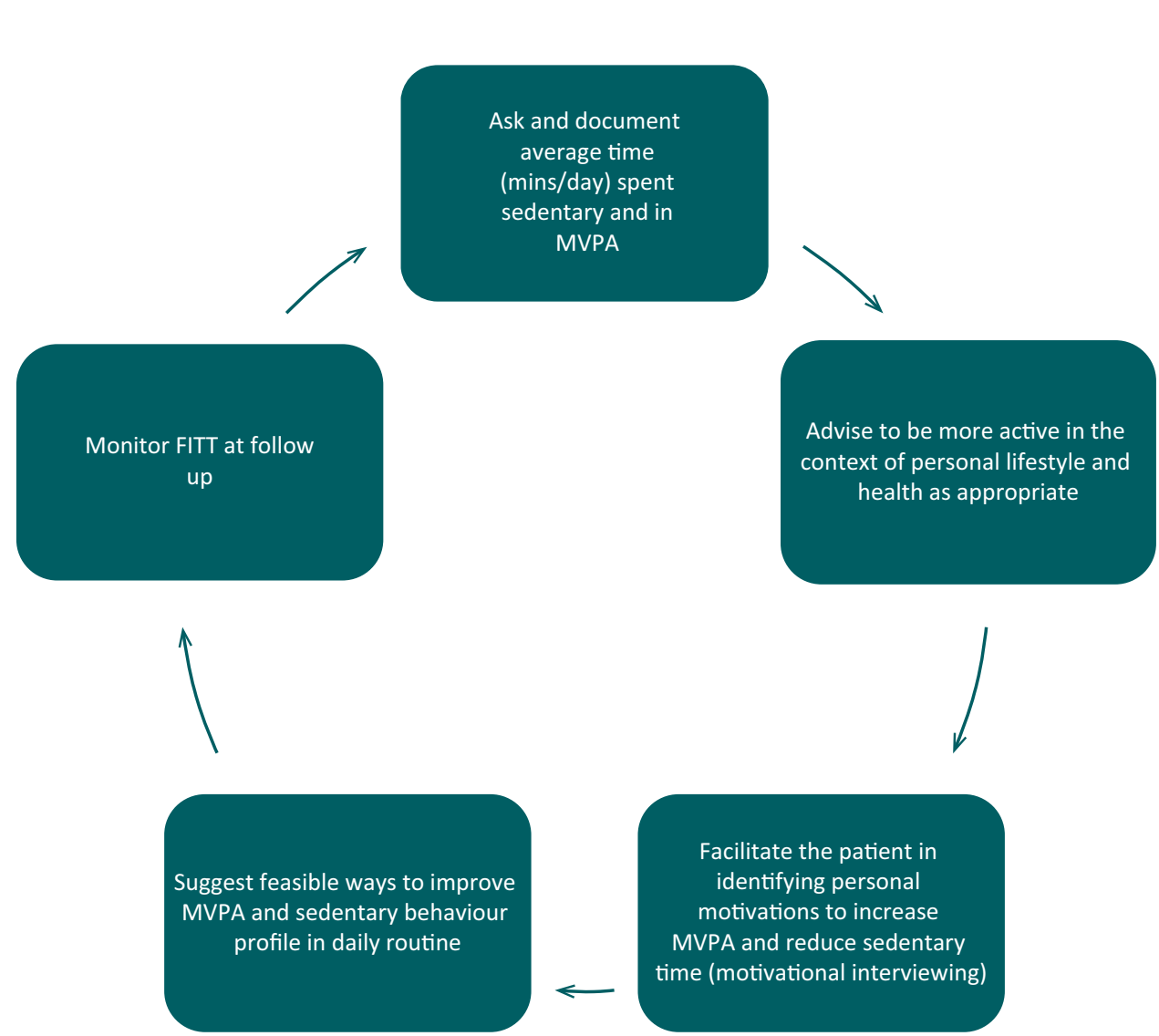

Fig 1. Incorporating PA into every consultation. MVPA = moderate and vigorous physical activity; $\mathrm{PA}=$ physical activity. 
Table 3. Key strategies for PA promotion.

\begin{tabular}{|c|c|c|}
\hline Aims & Tips & Practical steps \\
\hline $\begin{array}{l}\text { Incorporate small increments into daily } \\
\text { routine }\end{array}$ & $\begin{array}{l}10 \text { minute moderate and vigorous PA bouts } \\
\text { are most beneficial to health, but light PA is } \\
\text { more beneficial than none at all }\end{array}$ & $\begin{array}{l}\text { > Active breaks at school or work to reduce } \\
\text { uninterrupted sedentary behavior } \\
>\text { Take the stairs } \\
>\text { Stand up from sitting and stretch every } \\
20-30 \text { minutes }\end{array}$ \\
\hline $\begin{array}{l}\text { Ascertain baseline activity levels and } \\
\text { measure intra-individual changes }\end{array}$ & $\begin{array}{l}\text { Remember the capacity for PA naturally } \\
\text { increases through maturation and growth }\end{array}$ & $\begin{array}{l}\text { > Involve and educate to enable self-efficacy } \\
\text { in PA management }\end{array}$ \\
\hline Encourage family-based activity & $\begin{array}{l}\text { Children of physically active parents are } \\
\text { more physically active than children of } \\
\text { inactive parents }^{22}\end{array}$ & > Involve family in management \\
\hline Advocate active transport & Promote PA as part of daily life & $\begin{array}{l}>\text { Encourage walking part way to school or work } \\
>\text { Use cycle routes }\end{array}$ \\
\hline Engage a wide range of stakeholders & $\begin{array}{l}\text { Support safe, sociable environments and } \\
\text { green spaces }\end{array}$ & $\begin{array}{l}\text { Include schools, parents and the local } \\
\text { authority } \\
\text { Signpost to useful web-based PA information } \\
\text { hubs, such as the local council or British Heart } \\
\text { Foundation }\end{array}$ \\
\hline Enable children to work together & Establish schools as physical activity hubs & $\begin{array}{l}\text { Encourage links between adolescents, } \\
\text { school, family and community }\end{array}$ \\
\hline Establish links to local services & Be aware of local referral pathways & > Consult NICE PA overview $2014^{23}$ \\
\hline
\end{tabular}

The case study in Box 3 exemplifies PA prescription for an adolescent, where PIA is impacting negatively upon health and wellbeing.

\section{The risks from physical activity}

Risks are very low compared with potential health benefits, but practitioner awareness is key. The general physician should enquire about:

$>$ a history of exercise-associated dizziness, presyncope or collapse

$>$ family history of sudden cardiac death, unexplained death or drowning, especially in those under 35

> comorbidities, for example asthma or diabetes, where additional specialist advice and initial monitoring may be required.

Commencing purposeful light PA and instigating sedentary behaviour reduction is very safe. Progressing to moderateintensity PA is safe in the vast majority of patients with well managed comorbidities, in the context of salient advice regarding safe self-management for exacerbation of their symptoms, when to seek help and the principle of gradual progression. In a small number of cases deemed higher risk, onward referral to a sport and exercise medicine physician or other appropriate specialist is recommended for formal screening and risk stratification.

\section{Implementing physical activity advice in practice: the} inactive and obese adolescent

The risk factors of PIA in adolescence are highly relevant to the case presented in Box 3 and are presented in Box 4. PA is
Box 3. Emma, 14, advised to see her doctor by the

school nurse concerned about Emma's weight gain and withdrawn behaviour.

Emma's growth has been mapped to the 90th percentile since age 10 . She lives with her mother and sister who are both overweight. Her father, 45, has metabolic syndrome. No one in the family attains recommended levels of PA. Emma skips breakfast, eats lunch at a fast-food outlet and a supermarket 'ready meal' in the evening. She accumulates six hours per day screen time. Emma expresses dissatisfaction in feeling 'unfit.'

a core component of the multidisciplinary National Institute for Health and Care Excellence (NICE) lifestyle change management approach for overweight and obese young people, with emphasis on education, nutrition and a supportive environment with family and school engagement. ${ }^{26}$ Qualified health professionals can advise on PA in a primary or secondary care setting, with attention to physical, psychological and physiological barriers. $^{27}$

Adolescents with complex obesity should be referred to tier 3 specialist obesity services. Bariatric surgery is reserved for severely morbidly obese adolescents (BMI $>50$ or $>40$ with comorbidity). Surgery is associated with significant risks, with a paucity of long-term outcome and safety data. Ethical concerns arise regarding invasive management of lifestyle-related conditions. ${ }^{28}$ 
Box 4. Health risks in adolescent obesity. ${ }^{24,25}$

Increased prevalence of insulin resistance in obese children

> Three-fold likelihood of obese adolescents developing type-2 diabetes

Higher risk in some ethnic minorities

Increased risk of bone and joint injury

Excess load on developing skeleton (eg tibia vara, slipped capital femoral epiphysis)

$>$ Higher self-reported musculoskeletal pain

Psychological health

> Higher prevalence of depression and lower self-esteem

$50-75 \%$ of obese adolescents become obese adults

Heightened risk of metabolic disease, cardiovascular and musculoskeletal problems

\section{The initial consultation}

$>$ Document PA as daily MET intensity and time equivalents.

$>$ Use principles of motivational interviewing (MI). ${ }^{29}$

$>$ Exclude contraindications to immediate commencement of PA or the need for screening.

> Agree a PA action plan and 'exercise prescription' including:

> initial and incremental steps, using the 'FITT' principle

$>$ preferred activity options

$>$ supportive influences eg named friends, family and pets

$>$ short-term goals for the next review appointment

$>$ long-term goals.

Eliciting the adolescent's preference for type of physical activity is vital. Home-based exercise may be preferable initially where self-confidence is low, with peer-supported group exercise introduced progressively. Behaviour change strategies can enhance self-efficacy and an online app is available to provide health professional skills in motivational interviewing. ${ }^{30}$ Initiating exercise at low intensity is appropriate for the least active and confers the greatest initial benefits. ${ }^{31}$

\section{Weight management}

Targeting $0.5-1 \mathrm{~kg}$ reduction per week for healthy weight loss should be supported by dietary advice regarding reduced calorific intake, while increasing energy expenditure through PA and breaking up sedentary time.

\section{Physical activity and mental health}

Promotion of psychological wellbeing is essential. Further concerns about mental health should be addressed in conjunction with mental health services.

\section{Physical activity and environmental factors}

Urban living in communities with multiple deprivations and limited access to safe open spaces has been shown to have detrimental health impact. ${ }^{5}$ Urban residential areas can present a hostile environment to psychological, physical and cognitive wellbeing, engendering chronic stress and isolation. ${ }^{32}$ There is a positive correlation between PA levels in young people where park and open-space play areas are available close to home and school, and where recreational infrastructure is improved.

\section{Transport}

The majority of school children commute an average two miles to school. Safety concerns, a lack of pavements and poor lighting can present barriers to active transport such as walking or cycling to school. Improved local transport planning is recommended by NICE to facilitate active transport as the number of physically active commuters increases. ${ }^{33}$

Walking to school incorporates a 'dose' of PA within daily routine, replacing a sedentary commute while increasing social interaction. It provides first steps toward an active lifestyle, from which point progression to moderate and vigorous PA, such as cycling, swimming and team sports, can be made.

\section{Conclusion}

PA promotion offers a preventative and therapeutic tool akin to a polypill that reduces morbidity, mortality and economic burden from lifestyle-related non-communicable diseases. Healthcare professionals caring for adolescents are in a unique position to engender lifelong behaviour change. We advocate asking about PA and sedentary behaviour at every patient encounter with appropriate promotion and sign-posting strategies.

\section{References}

1 Lee IM, Shiroma EJ, Lobelo F et al. Effect of physical inactivity on major non-communicable diseases worldwide: an analysis of burden of disease and life expectancy. Lancet 2012;380:219-9.

2 Donaldson L. Annual report of the Chief Medical Officer 2010. London: DH, 2009.

3 Ruiz J, Castro-Pinero J, Arteo EG et al. Predictive validity of healthrelated fitness in youth: a systematic review. Br J Sports Med 2009;43:909-23.

4 Department of Health. Start active, stay active: a report on physical activity from the four home countries. London: DH, 2011.

5 Health and Social Care Information Centre. Health survey for England 2012. Leeds: Health and Social Care Information Centre, 2012.

6 StreetGames/Centre for Economic and Business Research. The inactivity timebomb. London: CEBR, 2014.

7 Brodersen N, Steptoe A. Trends in physical activity and sedentary behaviour in adolescence: ethnic and socioeconomic differences. Br J Sports Med 2007;41:140-4.

8 van Jaarsveld CHM, Gulliford MC. Childhood obesity trends from primary care electronic health records in England between 1994 and 2013: population-based cohort study. Arch Dis Child 2015;100:214-9.

9 Must S. Risks and consequences of childhood and adolescent obesity. Int J Obes Relat Metab Disord 1999;23S:2-11.

10 Gunnell D, Frankel S, Nanchahal K et al. Childhood obesity and adult cardiovascular mortality: a 57-year follow-up study based on the Boyd Orr Cohort. Am J Clin Nutr 1998;67:1111-8.

11 Blair SN. Physical inactivity: the biggest public health problem of the 21st century. Br J Sports Med 2009;43:1-2.

12 Department of Health. Healthy lives, healthy people: A call to action on obesity in England. London: DH, 2011.

13 Public Health England. Everybody active, everyday: An evidence based approach to physical activity. London: PHE, 2014. 
14 Durnin JVGA. Physical Activity and Health (Society for the Study of Human Biology Symposium Series). Cambridge: Cambridge University Press, 1992: 20-7.

15 Viner RM, Cole TJ. Television viewing in early childhood predicts adult body mass index. J Pediatr 2005;147:429-5.

16 Shiroma EJ, Lee IM. Exercise in cardiovascular disease, physical activity and cardiovascular health, lessons learned from epidemiological studies across age, gender, and race/ethnicity. Circulation 2010;122:743-52.

17 Santos R, Mota J, Okley A et al. The independent associations of sedentary behaviour and physical activity on cardiorespiratory fitness. Br J Sports Med 2014;48:1508-12.

18 Sibley BA, Etnier JL. The relationship between physical activity and cognition in children: A meta-analysis. Pediatr Exerc Sci 2003;15:243-56.

19 Davies S. Chief medical officer's annual report 2012: our children deserve better: prevention pays. London: DH, 2013.

20 Currie C, Zanotti C, Morgan A et al. Social determinants of health and wellbeing among young people. Health Behaviours in Schools Aged Children (HBSC) study: International report from the 2009/2010 survey. Geneva: WHO, 2010.

21 de Souto Barreto P. Global health agenda on non-communicable diseases: has WHO set a smart goal for physical activity? BMJ 2015;350:h23.

22 Jago R, Fox K, Page A, Brockman R, Thompson JL. Parent and child physical activity and sedentary time: Do active parents foster active children? BMC Public Health 2010;10:194.

23 National Institute for Health and Care Excellence. Physical activity overview. London: NICE, 2014.

24 Haines L, Wan KC, Lynn R, Barrett TG, Shield JPH. Rising incidence of type 2 diabetes in children in the U.K. Diabetes Care 2007;30:1097-101.

25 Krul M, van der Wouden J, Schellevis F, van Suijlekom-Smit 1, Koes B. Musculoskeletal problems in overweight and obese children. Ann Fam Med 2009;7:352-6.
26 National Institute of Health and Care Excellence. Managing overweight and obesity among children and young people: lifestyle weight management services. London: NICE, 2014.

27 Schranz N, Tomkinson G, Parletta N, Petov J, Olds T. Can resistance training change the strength, body composition and selfconcept of overweight and obese adolescent males? A randomised controlled trial. Br J Sports Med 2014;48:1482-8.

28 Hofmann B. Bariatric surgery for obese children and adolescents: a review of the moral challenges. BMC Medical Ethics 2013;14.

29 Resnicow K, Bocian A, Harris D et al. Can brief motivational interviewing in practice reduce child body mass index? Results of a 2-year randomized controlled trial. Elk Grove Village, IL: AAP, 2014. Available online at www.aap.org/en-us/professional-resources/Research/ research-findings/Pages/Can-Brief-Motivational-Interviewing-inPractice-Reduce-Child-Body-Mass-Index-Results-of-a-2-yearRandomized-Controlled-Tria.aspx [Accessed 6 March 2015].

30 American Academy of Paediatrics. Change Talk: Childhood Obesity. Elk Grove Village, IL: AAP Institute for Healthy Childhood Weight, 2014. Available online at http://ihcw.aap.org/resources [Accessed 6 March 2015].

31 Department of Health. At least five a week: evidence on the impact of physical activity and its relationship to health-A report from the chief medical officer. London: DH, 2004.

32 Davidson K, Lawson C. Do attributes in the physical environment influence children's level of physical activity? Int J Behav Nutr Phys Act 2006;3:1-17.

33 National Institute for Health and Care Excellence. Walking and cycling: local measures to promote walking and cycling as forms of travel or recreation. London: NICE, 2012.

Address for correspondence: Dr B Kumar, Institute of Sport Exercise and Health, University College London, 170 Tottenham Court Road, London W1T 7HA, UK. Email: bhavesh.kumar@ucl.ac.uk

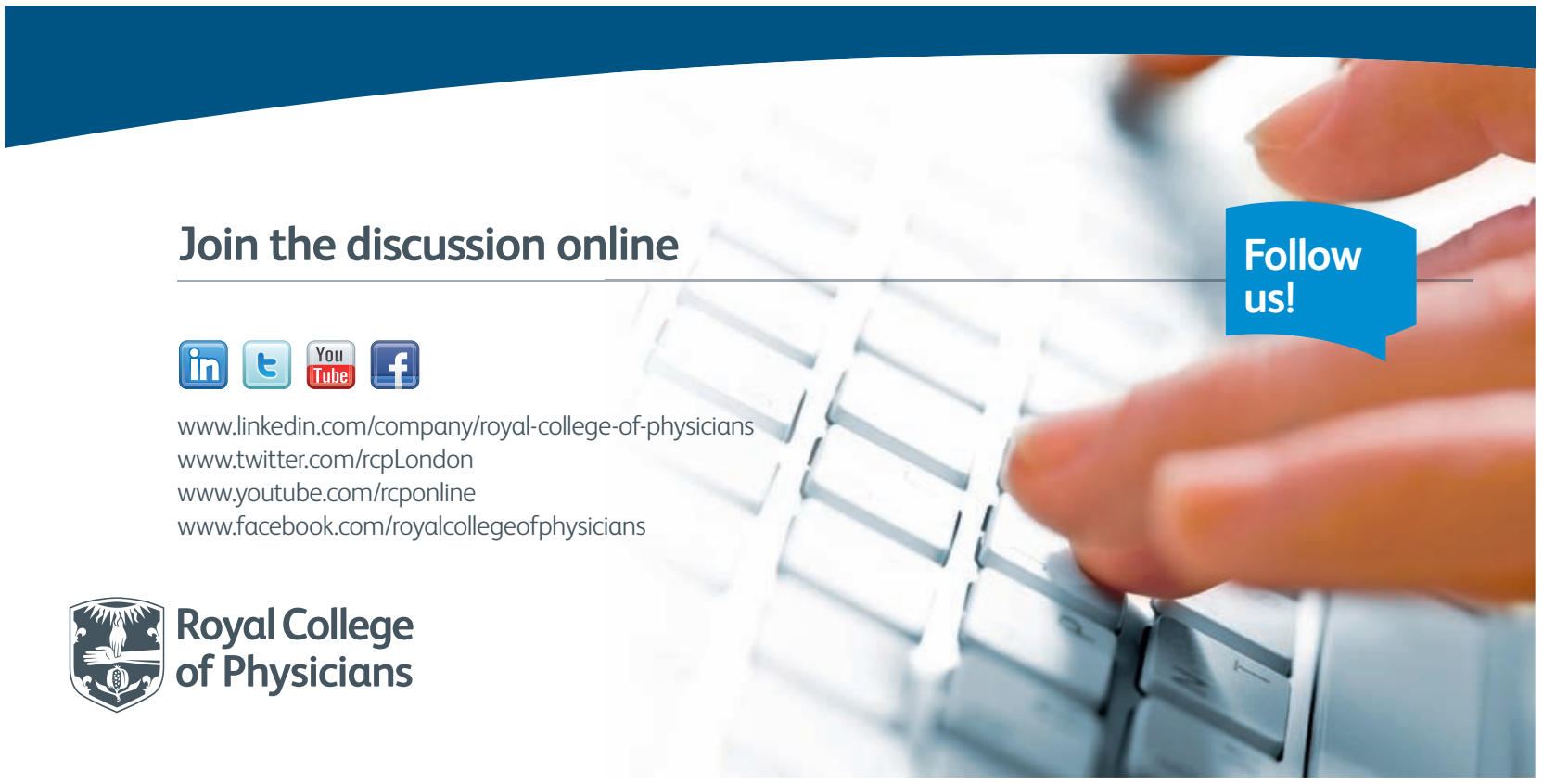

